\title{
Neural Naturalist: Generating Fine-Grained Image Comparisons
}

\author{
Maxwell Forbes Christine Kaeser-Chen Piyush Sharma Serge Belongie \\ University of Washington Google Research Cornell University and Cornell Tech \\ mbforbes @cs. washington.edu \\ \{christinech, piyushsharma\}@google.com \\ sjb344@cornell. edu
}

https://mbforbes.github.io/neural-naturalist/

\begin{abstract}
We introduce the new Birds-to-Words dataset of $41 \mathrm{k}$ sentences describing fine-grained differences between photographs of birds. The language collected is highly detailed, while remaining understandable to the everyday observer (e.g., "heart-shaped face," "squat body"). Paragraph-length descriptions naturally adapt to varying levels of taxonomic and visual distance-drawn from a novel stratified sampling approach-with the appropriate level of detail. We propose a new model called Neural Naturalist that uses a joint image encoding and comparative module to generate comparative language, and evaluate the results with humans who must use the descriptions to distinguish real images.

Our results indicate promising potential for neural models to explain differences in visual embedding space using natural language, as well as a concrete path for machine learning to aid citizen scientists in their effort to preserve biodiversity.
\end{abstract}

\section{Introduction}

Humans are adept at making fine-grained comparisons, but sometimes require aid in distinguishing visually similar classes. Take, for example, a citizen science effort like iNaturalist, ${ }^{1}$ where everyday people photograph wildlife, and the community reaches a consensus on the taxonomic label for each instance. Many species are visually similar (e.g., Figure 1, top), making them difficult for a casual observer to label correctly. This puts an undue strain on lieutenants of the citizen science community to curate and justify labels for a large number of instances. While everyone may be capable of making such distinctions visually, nonexperts require training to know what to look for.

\footnotetext{
Work done during an internship at Google.

${ }^{1}$ https://www.inaturalist.org
}
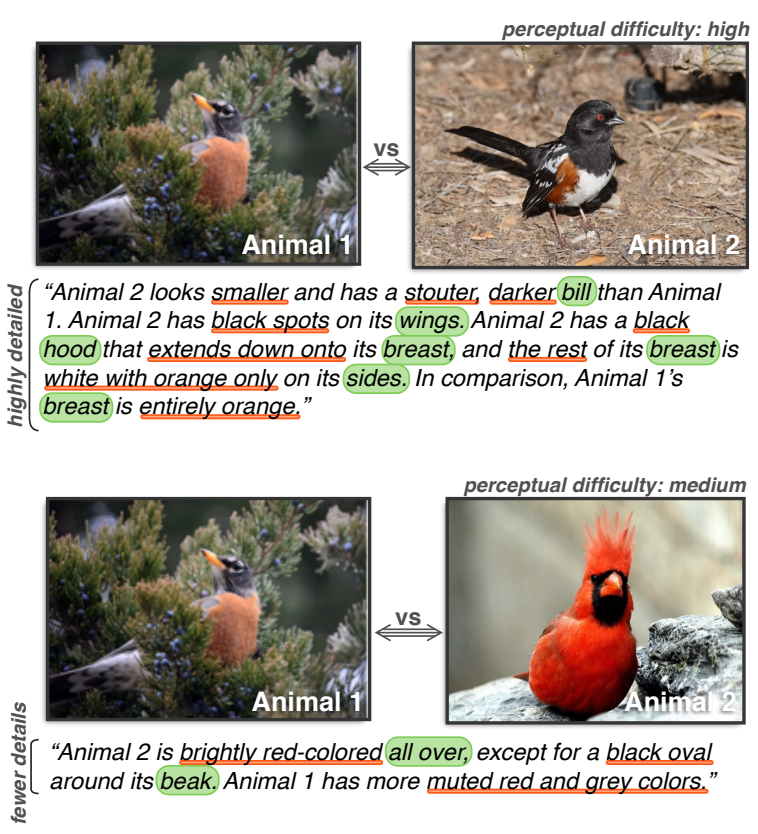

descriptive phrase body part

Figure 1: The Birds-to-Words dataset: comparative descriptions adapt naturally to the appropriate level of detail (orange underlines). A difficult distinction (TOP) is given a longer and more fined-grained comparison than an easier one (ВОТТОМ). Annotators organically use everyday language to refer to parts (green highlights).

Field guides exist for the purpose helping people learn how to distinguish between species. Unfortunately, field guides are costly to create because writing such a guide requires expert knowledge of class-level distinctions.

In this paper, we study the problem of explaining the differences between two images using natural language. We introduce a new dataset called Birds-to-Words of paragraph-length descriptions of the differences between pairs of bird photographs. We find several benefits from eliciting comparisons: (a) without a guide, annotators naturally break down the subject of the image (e.g., 
a bird) into pieces understood by the everyday observer (e.g., head, wings, legs); (b) by sampling comparisons from varying visual and taxonomic distances, the language exhibits naturally adaptive granularity of detail based on the distinctions required (e.g., "red body" vs "tiny stripe above its eye"); (c) in contrast to requiring comparisons between categories (e.g., comparing one species vs. another), non-experts can provide high-quality annotations without needing domain expertise.

We also propose the Neural Naturalist model architecture for generating comparisons given two images as input. After embedding images into a latent space with a $\mathrm{CNN}$, the model combines the two image representations with a joint encoding and comparative module before passing them to a Transformer decoder. We find that introducing a comparative module - an additional Transformer encoder-over the combined latent image representations yields better generations.

Our results suggest that these classes of neural models can assist in fine-grained visual domains when humans require aid to distinguish closely related instances. Non-experts-such as amateur naturalists trying to tell apart two species-stand to benefit from comparative explanations. Our work approaches this sweet-spot of visual expertise, where any two in-domain images can be compared, and the language is detailed, adaptive to the types of differences observed, and still understandable by laypeople.

Recent work has made impressive progress on context sensitive image captioning. One direction of work uses class labels as context, with the objective of generating captions that distinguish why the image belongs to one class over others (Hendricks et al., 2016; Vedantam et al., 2017). Another choice is to use a second image as context, and generate a caption that distinguishes one image from another. Previous work has studied ways to generalize single-image captions into comparative language (Vedantam et al., 2017), as well as comparing two images with high pixel overlap (e.g., surveillance footage) (Jhamtani and BergKirkpatrick, 2018). Our work complements these efforts by studying directly comparative, everyday language on image pairs with no pixel overlap.

Our approach outlines a new way for models to aid humans in making visual distinctions. The Neural Naturalist model requires two instances as input; these could be, for example, a query image

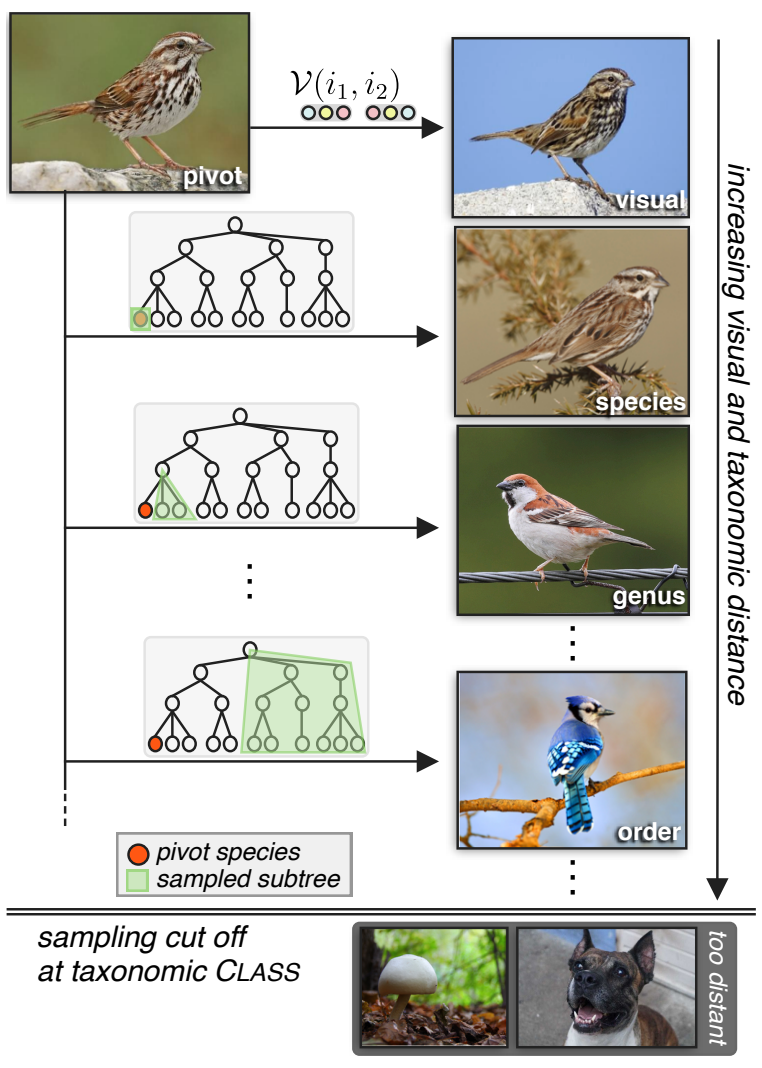

Figure 2: Illustration of pivot-branch stratified sampling algorithm used to construct the Birds-toWords dataset. The algorithm harnesses visual and taxonomic distances (increasing vertically) to create a challenging task with board coverage.

and an image from a candidate class. By differentiating between these two inputs, a model may help point out subtle distinctions (e.g., one animal has spots on its side), or features that indicate a good match (e.g., only a slight difference in size). These explanations can aid in understanding both differences between species, as well as variance within instances of a single species.

\section{Birds-to-Words Dataset}

Our goal is to collect a dataset of tuples $\left(i_{1}, i_{2}, t\right)$, where $i_{1}$ and $i_{2}$ are images, and $t$ is a natural language comparison between the two. Given a domain $\mathcal{D}$, this collection depends critically on the criteria we use to select image pairs.

If we sample image pairs uniformly at random, we will end up with comparisons encompassing a broad range of phenomena. For example, two images that are quite different will yield categorical comparisons ("One is a bird, one is a mushroom."). Alternatively, if the two images are very similar, such as two angles of the same creature, 


\begin{tabular}{llllll}
\hline Dataset & Domain & Lang & Ctx & Cap & Example \\
\hline $\begin{array}{l}\text { CUB Captions } \\
(R, 2016)\end{array}$ & Birds & M & 1 & 1 & $\begin{array}{l}\text { "An all black bird with a very long rectrices and relatively dull } \\
\text { bill." }\end{array}$ \\
\hline $\begin{array}{l}\text { CUB-Justify } \\
(V, 2017)\end{array}$ & Birds & $\mathrm{S}$ & 7 & 1 & $\begin{array}{l}\text { "The bird has white orbital feathers, a black crown, and yellow } \\
\text { tertials." }\end{array}$ \\
\hline $\begin{array}{l}\text { Spot-the-Diff } \\
(J \& B, 2018)\end{array}$ & Surveilance & $\mathrm{E}$ & 2 & $1-2$ & $\begin{array}{l}\text { "Silver car is gone. Person in a white t shirt appears. 3rd person } \\
\text { in the group is gone." }\end{array}$ \\
\hline $\begin{array}{l}\text { Birds-to-Words } \\
(\text { this work })\end{array}$ & Birds & $\mathrm{E}$ & 2 & 2 & $\begin{array}{l}\text { "Animall is gray, while animal2 is white. Animal2 has a long, } \\
\text { yellow beak, while animall's beak is shorter and gray. Animal2 } \\
\text { appears to be larger than animall." }\end{array}$ \\
\hline
\end{tabular}

Table 1: Comparison with recent fine-grained language-and-vision datasets. Lang values: $\mathrm{S}=$ scientific, $\mathrm{E}=$ everyday, $\mathrm{M}=$ mixed. Images $C t x=$ number of images shown, Images $C a p=$ number of images described in caption. Dataset citations: $R=$ Reed et al., $V=$ Vedantam et al., $J \& B=$ Jhamtani and Berg-Kirkpatrick.

comparisons between them will focus on highly detailed nuances, such as variations in pose. These phenomena support rich lines of research, such as object classification (Deng et al., 2009) and pose estimation (Murphy-Chutorian and Trivedi, 2009).

We aim to land somewhere in the middle. We wish to consider sets of distinguishable but intimately related pairs. This sweet spot of visual similarity is akin to the genre of differences studied in fine-grained visual classification (Wah et al., 2011; Krause et al., 2013). We approach this collection with a two-phase data sampling procedure. We first select pivot images by sampling from our full domain uniformly at random. We then branch from these images into a set of secondary images that emphases fine-grained comparisons, but yields broad coverage over the set of sensible relations. Figure 2 provides an illustration of our sampling procedure.

\subsection{Domain}

We sample images from iNaturalist, a citizen science effort to collect research-grade ${ }^{2}$ observations of plants and animals in the wild. We restrict our domain $\mathcal{D}$ to instances labeled under the taxonomic CLASS ${ }^{3}$ Aves (i.e., birds). While a broader domain would yield some comparable instances (e.g., bird and dragonfly share some common body parts), choosing only Aves ensures that all instances will be similar enough structurally to be comparable, and avoids the gut reaction compar-

\footnotetext{
${ }^{2}$ Research-grade observations have met or exceeded iNaturalist's guidelines for community consensus of the taxonomic label for a photograph.

${ }^{3}$ To disambiguate class, we use CLASS to denote the taxonomic rank in scientific classification, and simply "class" to refer to the machine learning usage of the term as a label in classification.
}

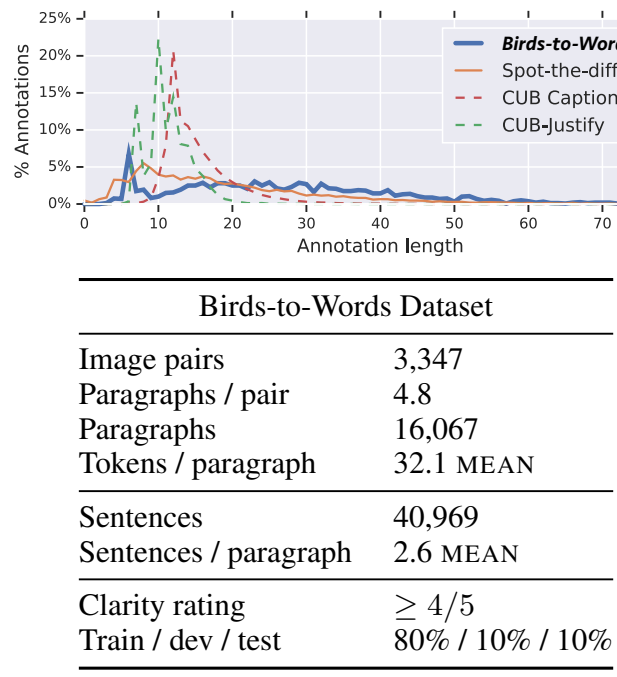

Figure 3: Annotation lengths for compared datasets (TOP), and statistics for the proposed Birds-toWords dataset (воттом). The Birds-to-Words dataset has a large mass of long descriptions in comparison to related datasets.

ison pointing out the differences in animal type. This choice yields $1.7 \mathrm{M}$ research-grade images and corresponding taxonomic labels from iNaturalist. We then perform pivot-branch sampling on this set to choose pairs for annotation.

\subsection{Pivot Images}

The Aves domain in iNaturalist contains instances of $9 \mathrm{k}$ distinct species, with heavy observation bias to more common species (such as the mallard duck). We uniformly sample species from the set of $9 \mathrm{k}$ to help overcome this bias. In total, we select 405 species and corresponding photographs to use as $i_{1}$ images. 


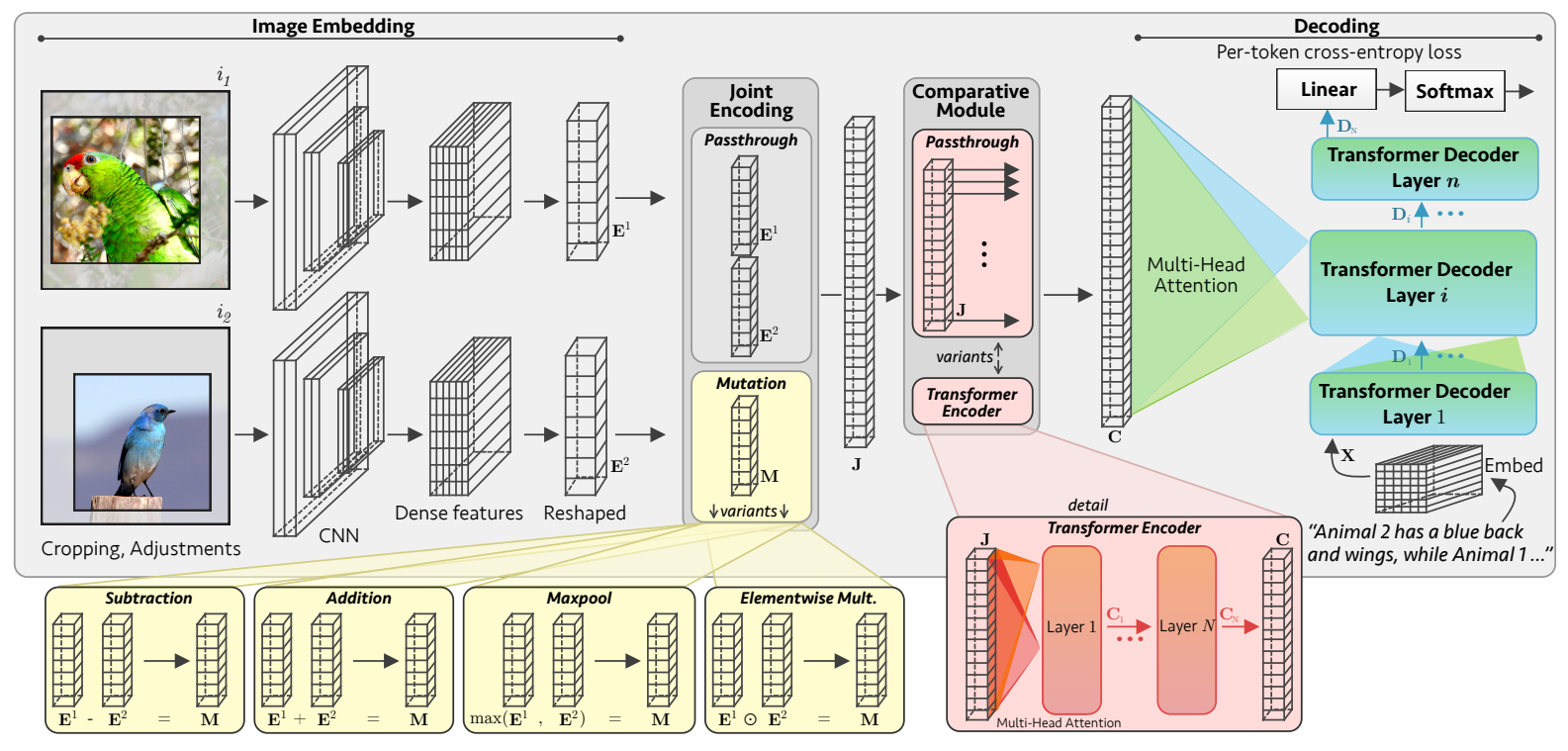

Figure 4: The proposed Neural Naturalist model architecture. The multiplicative joint encoding and Transformerbased comparative module yield the best comparisons between images.

\subsection{Branching Images}

We use both a visual similarity measure and taxonomy to sample a set of comparison images $i_{2}$ branching off from each pivot image $i_{1}$. We use a branching factor of $k=12$ from each pivot image.

To capture visually similar images to $i_{1}$, we employ a similarity function $\mathcal{V}\left(i_{1}, i_{2}\right)$. We use an Inception-v4 (Szegedy et al., 2017) network pretrained on ImageNet (Deng et al., 2009) and then fine-tuned to perform species classification on all research-grade observations in iNaturalist. We take the embedding for each image from the last layer of the network before the final softmax. We perform a k-nearest neighbor search by quantizing each embedding and using $\mathrm{L} 2$ distance (Wu et al., 2017; Guo et al., 2016), selecting the $k_{v}=2$ closest images in embedding space.

We also use the iNaturalist scientific taxonomy $\mathcal{T}(\mathcal{D})$ to sample images at varying levels of taxonomic distance from $i_{1}$. We select $k_{t}=10$ taxonomically branched images by sampling two images each from the same $\operatorname{SPECIES}(\ell=1)$, GENUS, FAMILY, ORDER, and CLASS $(\ell=5)$ as $c$. This yields 4,860 raw image pairs $\left(i_{1}, i_{2}\right)$.

\subsection{Language Collection}

For each image pair $\left(i_{1}, i_{2}\right)$, we elicit five natural language paragraphs describing the differences between them.

An annotator is instructed to write a paragraph (usually $2-5$ sentences) comparing and contrasting the animal appearing in each image. We instruct annotators not to explicitly mention the species (e.g., "Animal 1 is a penguin"), and to instead focus on visual details (e.g., "Animal 1 has a black body and a white belly"). They are additionally instructed to avoid mentioning aspects of the background, scenery, or pose captured in the photograph (e.g., "Animal 2 is perched on a coconut").

We discard all annotations for an image pair where either image did not have at least $\frac{4}{5}$ positive ratings of image clarity. This yields a total of 3,347 image pairs, annotated with 16,067 paragraphs. Detailed statistics of the Birds-toWords dataset are shown in Figure 3, and examples are provided in Figure 5. Further details of our both our algorithmic approach and dataset construction are given in Appendices A and B.

\section{Neural Naturalist Model}

Task Given two images $\left(i_{1}, i_{2}\right)$ as input, our task is to generate a natural language paragraph $t=x_{1} \ldots x_{n}$ that compares the two images.

Architecture Recent image captioning approaches (Xu et al., 2015; Sharma et al., 2018) extract image features using a convolutional neural network (CNN) which serve as input to a language decoder, typically a recurrent neural network (RNN) (Mikolov et al., 2010) or Transformer (Vaswani et al., 2017). We extend this paradigm with a joint encoding step and comparative module to study how best to encode and transform 


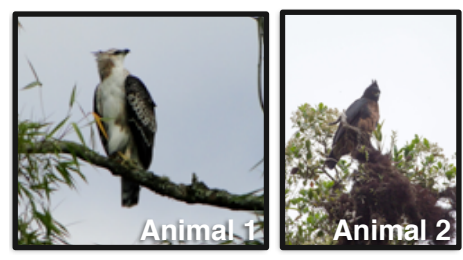

$\mathrm{M}$ animal1 is brown and white with a shor long gray beak

C animal1 is white with dark brown and white wings and a golden head . animal2 is brown-gold with dark solid-colored brown wings and a dark head.
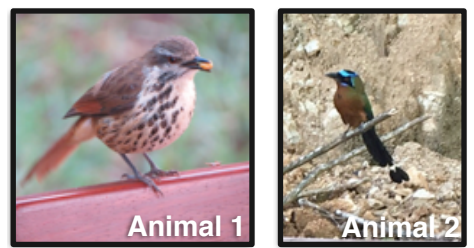

animal1 is two - toned brown with a white patch on its head . animal2 is multi - colored with longer tail feathers

animal1 is brown and white with a squatty body with a light brown head animal2 is multi - colored with a light blue and black head
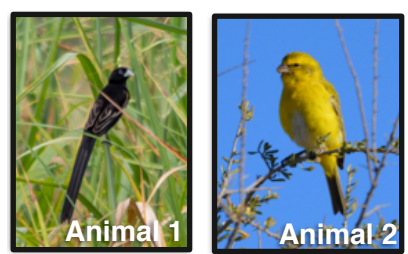

nimal1 is white with brown wing while animal2 is yellow with black head

animal1 has a long dark tail and

flecked dark wings with a white

curved beak . animal2 has a shorter beak and a yellow breast and head with a shorter brown tail

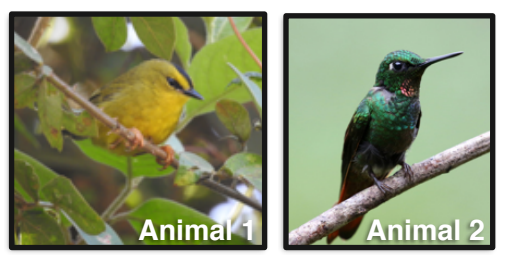

$\mathbf{M}$ animal1 is a dull yellow with grey tail feathers while animal2 is a yellow-green

animal1 has dark orange claws, while animal2 has grey claws . animal1 has yellow coloring with black on the top of the head and in tiny wing patches, animal2 is mostly green with red on the neck and brown on the wings .
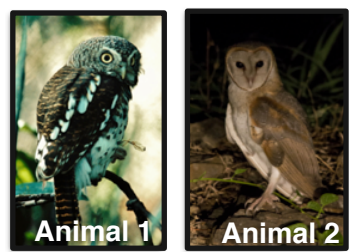

$M$

animal2 has a heart shaped face, whereas animal1 has an oval face. animal2 has entirely dark eyes. animal2 has a white beak, whereas animal1 has a dark beak . animal2 has more white in its feathers

animal1 has yellow eyes . animal2 has black eyes animal2 is lighter in color than animal1 . animal has a heart shaped face . animall doesn ' $t$.

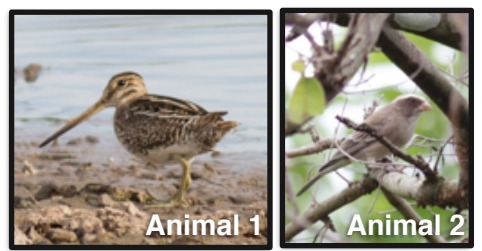

M

animal1 is brown with black spots on th body while animal2 is tan with a white neck and black head

G animal1 is brown and white with a long yellow and brown beak . animal2 is gray with a short light pink beak.
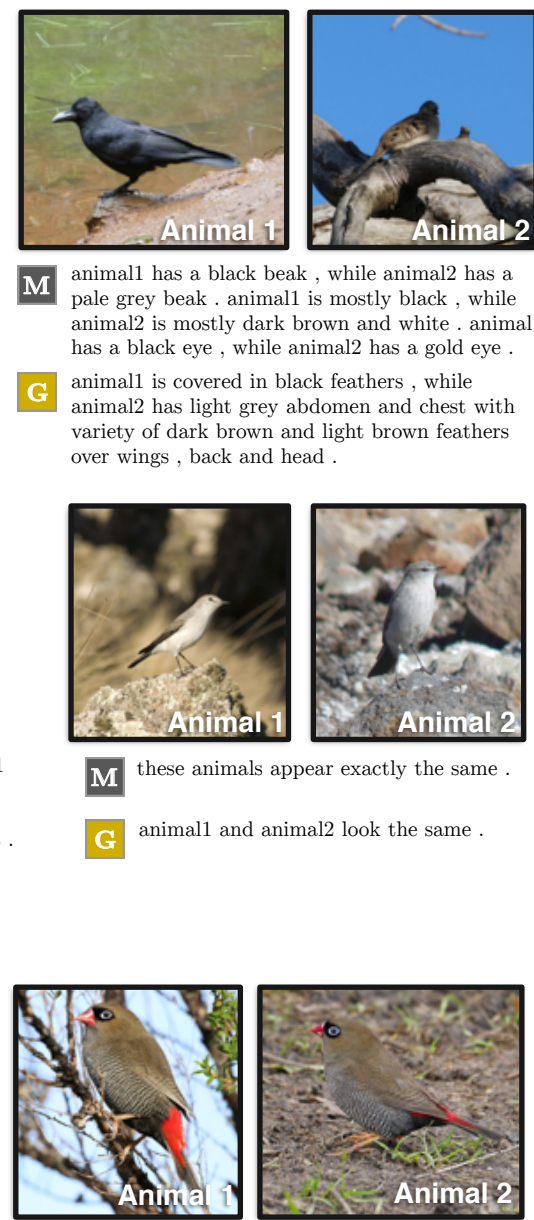

M animal1 has a black beak, while animal2 has a pale grey beak . animal1 is mostly black, while animal2 is mostly dark brown and white . animal has a black eye, while animal2 has a gold eye

G animal1 is covered in black feathers, while animal2 has light grey abdomen and chest with variety of dark brown and light brown feathers over wings, back and head.

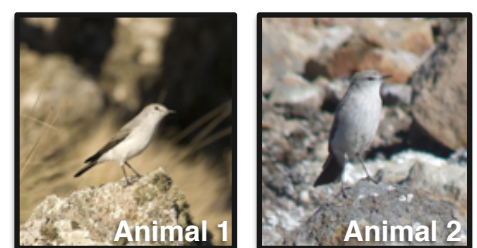

$\mathbf{M}$ these animals appear exactly the same.

G animal1 and animal2 look the same.

M

nimal2 ' s colors are brighter than animal1 animal2 has more earthy colors than animal1 animal1 is a bit bigger than animal2

G both animals appear to be the same

Figure 5: Samples from the dev split of the proposed Birds-to-Words dataset, along with Neural Naturalist model output (M) and one of five ground truth paragraphs $(\mathrm{G})$. The second row highlights failure cases in red. The model produces coherent descriptions of variable granularity, though emphasis and assignment can be improved.

multiple latent image embeddings. A schematic of the model is outlined in Figure 4, and its key components are described in the upcoming sections.

\subsection{Image Embedding}

Both input images are first processed using CNNs with shared weights. In this work, we consider ResNet (He et al., 2016) and Inception (Szegedy et al., 2017) architectures. In both cases, we extract the representation from the deepest layer immediately before the classification layer. This yields a dense $2 \mathrm{D}$ grid of local image feature vectors, shaped $(d, d, f)$. We then flatten each feature grid into a $\left(d^{2}, f\right)$ shaped matrix:

$$
\begin{aligned}
& \mathbf{E}^{1}=\left\langle\mathbf{e}_{1,1}^{1}, \ldots, \mathbf{e}_{d, d}^{1}\right\rangle=\operatorname{CNN}\left(i_{1}\right) \\
& \mathbf{E}^{2}=\left\langle\mathbf{e}_{1,1}^{2}, \ldots, \mathbf{e}_{d, d}^{2}\right\rangle=\operatorname{CNN}\left(i_{2}\right)
\end{aligned}
$$

\subsection{Joint Encoding}

We define a joint encoding $\mathbf{J}$ of the images which contains both embedded images $\left(\mathbf{E}^{1}, \mathbf{E}^{2}\right)$, a mutated combination $(\mathbf{M})$, or both. We consider as possible mutations $\mathbf{M} \in\left\{\mathbf{E}^{1}+\mathbf{E}^{2}, \mathbf{E}^{1}-\right.$ $\left.\mathbf{E}^{2}, \max \left(\mathbf{E}^{1}, \mathbf{E}^{2}\right), \mathbf{E}^{1} \odot \mathbf{E}^{2}\right\}$. We try these encoding variants to explore whether simple mutations can effectively combine the image representations. 


\subsection{Comparative Module}

Given the joint encoding of the images $(\mathbf{J})$, we would like to represent the differences in feature space (C) in order to generate comparative descriptions. We explore two variants at this stage. The first is a direct passthrough of the joint encoding $(\mathbf{C}=\mathbf{J})$. This is analogous to "standard" CNN+LSTM architectures, which embed images and pass them directly to an LSTM for decoding. Because we try different joint encodings, a passthrough here also allows us to study their effects in isolation.

Our second variant is an $N$-layer Transformer encoder. This provides an additional self-attentive mutations over the latent representations $\mathbf{J}$. Each layer contains a multi-headed attention mechanism $\left(\mathrm{ATTN}_{\mathrm{MH}}\right)$. The intent is that self-attention in Transformer encoder layers will guide comparisons across the joint image encoding.

Denoting LN as Layer Norm and FF as Feed Forward, with $\mathbf{C}_{i}$ as the output of the $i$ th layer of the Transformer encoder, $\mathbf{C}_{0}=\mathbf{J}$, and $\mathbf{C}=\mathbf{C}_{N}$ :

$$
\begin{aligned}
\mathbf{C}_{i}^{H} & =\mathrm{LN}\left(\mathbf{C}_{i-1}+\operatorname{ATTN}_{\mathrm{MH}}\left(\mathbf{C}_{i-1}\right)\right) \\
\mathbf{C}_{i} & =\mathrm{LN}\left(\mathbf{C}_{i}^{H}+\operatorname{FF}\left(\mathbf{C}_{i}^{H}\right)\right)
\end{aligned}
$$

\subsection{Decoder}

We use an $N$-layer Transformer decoder architecture to produce distributions over output tokens. The Transformer decoder is similar to an encoder, but it contains an intermediary multi-headed attention which has access to the encoder's output $\mathbf{C}$ at every time step.

$$
\begin{aligned}
\mathbf{D}_{i}^{H_{1}} & =\mathrm{LN}\left(\mathbf{X}+\operatorname{ATTN}_{\mathrm{MASK}, \mathrm{MH}}(\mathbf{X})\right) \\
\mathbf{D}_{i}^{H_{2}} & =\mathrm{LN}\left(\mathbf{D}_{i}^{H_{1}}+\operatorname{ATTN}_{\mathrm{MH}}\left(\mathbf{D}_{i}^{H_{1}}, \mathbf{C}\right)\right) \\
\mathbf{D}_{i} & =\mathrm{LN}\left(\mathbf{D}_{i}^{H_{2}}+\operatorname{FF}\left(\mathbf{D}_{i}^{H_{2}}\right)\right)
\end{aligned}
$$

Here we denote the text observed during training as $\mathbf{X}$, which is modulated with a position-based encoding and masked in the first multi-headed attention.

\section{Experiments}

We train the Neural Naturalist model to produce descriptions of the differences between images in the Birds-to-Words dataset. We partition the dataset into train (80\%), val (10\%), and test (10\%) sections by splitting based on the pivot images $i_{1}$.
This ensures $i_{1}$ species are unique across the different splits.

We provide model hyperparameters and optimization details in Appendix C.

\subsection{Baselines and Variants}

The most frequent paragraph baseline produces only the most observed description in the training data, which is that the two animals appear to be exactly the same. Text-Only samples captions from the training data according to their empirical distribution. Nearest Neighbor embeds both images and computes the lowest total $L_{2}$ distance to a training set pair, sampling a caption from it. We include two standard neural baselines, CNN (+ Attention) + LSTM, which concatenate the images embeddings, optionally perform attention, and decode with an LSTM. The main model variants we consider are a simple joint encoding $\left(\mathbf{J}=\left\langle\mathbf{E}^{1}, \mathbf{E}^{2}\right\rangle\right)$, no comparative module $(\mathbf{C}=\mathbf{J})$, a small (1-layer) decoder, and our full Neural Naturalist model. We also try several other ablations and model variants, which we describe later.

\subsection{Quantitative Results}

Automatic Metrics We evaluate our model using three machine-graded text metrics: BLEU4 (Papineni et al., 2002), ROUGE-L (Lin, 2004), and CIDEr-D (Vedantam et al., 2015). Each generated paragraph is compared to all five reference paragraphs.

For human performance, we use a one-vs-rest scheme to hold one reference paragraph out and compute its metric using the other four. We average this score across twenty-five runs over the entire split in question.

Results using these metrics are given in Table 2 for the main baselines and model variants. We observe improvement across BLEU-4 and ROUGEL scores compared to baselines. Curiously, we observe that the CIDEr-D metric is susceptible to common patterns in the data; our model, when stopped at its highest CIDEr-D score, outputs a variant of, "these animals appear exactly the same" for $95 \%$ of paragraphs, nearly mimicking the behavior of the most frequent paragraph (Freq.) baseline. The corpus-level behavior of CIDEr-D gives these outputs a higher score. We observed anecdotally higher quality outputs correlated with ROUGE-L score, which we verify using a human evaluation (paragraph after next). 


\begin{tabular}{|c|c|c|c|c|c|c|}
\hline & \multicolumn{3}{|c|}{ Dev } & \multicolumn{3}{|c|}{ Test } \\
\hline & BLEU-4 & ROUGE-L & CIDEr-D & BLEU-4 & ROUGE-L & CIDEr-D \\
\hline Most Frequent & 0.20 & 0.31 & 0.42 & 0.20 & 0.30 & 0.43 \\
\hline Text-Only & 0.14 & 0.36 & 0.05 & 0.14 & 0.36 & 0.07 \\
\hline Nearest Neighbor & 0.18 & 0.40 & 0.15 & 0.14 & 0.36 & 0.06 \\
\hline CNN + LSTM (Vinyals et al., 2015) & 0.22 & 0.40 & 0.13 & 0.20 & 0.37 & 0.07 \\
\hline CNN + Attn. + LSTM (Xu et al., 2015) & 0.21 & 0.40 & 0.14 & 0.19 & 0.38 & 0.11 \\
\hline Neural Naturalist - Simple Joint Encoding & 0.23 & 0.44 & 0.23 & - & - & - \\
\hline Neural Naturalist - No Comparative Module & 0.09 & 0.27 & 0.09 & - & - & - \\
\hline Neural Naturalist - Small Decoder & 0.22 & 0.42 & 0.25 & - & - & - \\
\hline Neural Naturalist - Full & 0.24 & 0.46 & 0.28 & 0.22 & 0.43 & 0.25 \\
\hline Human & $0.26+/-0.02$ & $0.47+/-0.01$ & $0.39+/-0.04$ & $0.27+/-0.01$ & $0.47+/-0.01$ & $0.42+/-0.03$ \\
\hline
\end{tabular}

Table 2: Experimental results for comparative paragraph generation on the proposed dataset. For human captions, mean and standard deviation are given for a one-vs-rest scheme across twenty-five runs. We observed that CIDErD scores had little correlation with description quality. The Neural Naturalist model benefits from a strong joint encoding and Transformer-based comparative module, achieving the highest BLEU-4 and ROUGE-L scores.

Ablations and Model Variants We ablate and vary each of the main model components, running the automatic metrics to study coarse changes in the model's behavior. Results for these experiments are given in Table 3. For the joint encoding, we try combinations of four element-wise operations with and without both encoded images. To study the comparative module in greater detail, we examine its effect on the top three joint encodings: $\left(i_{1}, i_{2},-\right),-$, and $\odot$. After fixing the best joint encoding and comparative module, we also try variations of the decoder (Transformer depth), as well as decoding algorithms (greedy decoding, multinomial sampling, and beamsearch).

Overall, we we see that the choice of joint encoding requires a balance with the choice of comparative module. More disruptive joint encodings (like element-wise multiplication $\odot$ ) appear too destructive when passed directly to a decoder, but yield the best performance when paired with a deep comparative module. Others (like subtraction) function moderately well on their own, and are further improved when a comparative module is introduced.

Human Evaluation To verify our observations about model quality and automatic metrics, we also perform a human evaluation of the generated paragraphs. We sample 120 instances from the test set, taking twenty each from the six categories for choosing comparative images (visual similarity in embedding space, plus five taxonomic distances). We provide annotators with the two images in a random order, along with the output from the model at hand. Annotators must decide which image contains Animal 1, and which contains Animal 2, or they may say that there is no way to tell (e.g., for a description like "both look exactly the same").

We collect three annotations per datum, and score a decision only if $\geq 2 / 3$ annotators made that choice. A model receives +1 point if annotators decide correctly, 0 if they cannot decide or agree there is no way to tell, and -1 point if they decide incorrectly (label the images backwards). This scheme penalizes a model for confidently writing incorrect descriptions. The total score is then normalized to the range $[-1,1]$. Note that Human uses one of the five gold paragraphs sampled at random.

Results for this experiment are shown in Table 4. In this measure, we see the frequency and text-only baselines now fall flat, as expected. The frequency baseline never receives any points, and the text-only baseline is often penalized for incorrectly guessing. Our model is successful at making distinctions between visually distinct species (GENUS column and ones further right), which is near the challenge level of current fine-grained visual classification tasks. However, it struggles on the two data subsets with highest visual similarity (VISUAL, SPECIES). The significant gap between all methods and human performance in these columns indicates ultra fine-grained distinctions are still possible for humans to describe, but pose a challenge for current models to capture.

\subsection{Qualitative Analysis}

In Figure 5, we present several examples of the model output for pairs of images in the dev set, along with one of the five reference paragraphs. In 


\begin{tabular}{|c|c|c|c|c|c|c|c|c|c|c|c|}
\hline \multicolumn{6}{|c|}{ Joint Encoding } & \multirow[b]{2}{*}{ Comparative Module } & \multirow[b]{2}{*}{ Decoder } & \multirow{2}{*}{$\begin{array}{l}\text { Decoding } \\
\text { Algorithm }\end{array}$} & \multicolumn{3}{|c|}{ Dev } \\
\hline$i_{1}$ & $i_{2}$ & - & + & $\max$ & $\odot$ & & & & BLEU-4 & ROUGE-L & CIDEr-D \\
\hline \multirow[t]{5}{*}{$\checkmark$} & $\checkmark$ & & & & & \multirow{10}{*}{$\begin{array}{c}\text { 6-Layer } \\
\text { Transformer }\end{array}$} & \multirow{10}{*}{$\begin{array}{c}\text { 6-Layer } \\
\text { Transformer }\end{array}$} & \multirow{10}{*}{ Beamsearch } & 0.23 & 0.44 & 0.23 \\
\hline & & $\checkmark$ & & & & & & & 0.23 & 0.45 & 0.27 \\
\hline & & & $\checkmark$ & & & & & & 0.24 & 0.43 & 0.28 \\
\hline & & & & $\checkmark$ & & & & & 0.23 & 0.43 & 0.24 \\
\hline & & & & & $\checkmark$ & & & & 0.24 & 0.46 & 0.28 \\
\hline$\checkmark$ & $\checkmark$ & $\checkmark$ & & & & & & & 0.22 & 0.44 & 0.22 \\
\hline$\checkmark$ & $\checkmark$ & & $\checkmark$ & & & & & & 0.22 & 0.42 & 0.25 \\
\hline$\checkmark$ & $\checkmark$ & & & $\checkmark$ & & & & & 0.21 & 0.42 & 0.22 \\
\hline$\checkmark$ & $\checkmark$ & & & & $\checkmark$ & & & & 0.22 & 0.43 & 0.23 \\
\hline \multirow[t]{9}{*}{$\checkmark$} & $\checkmark$ & $\checkmark$ & $\checkmark$ & $\checkmark$ & $\checkmark$ & & & & 0.21 & 0.43 & 0.20 \\
\hline & & & & & $\checkmark$ & Passthrough & \multirow{4}{*}{$\begin{array}{c}\text { 6-Layer } \\
\text { Transformer }\end{array}$} & \multirow{4}{*}{ Beamsearch } & 0.00 & 0.02 & 0.00 \\
\hline & & & & & $\checkmark$ & 1-L Transformer & & & 0.24 & 0.44 & 0.27 \\
\hline & & & & & $\checkmark$ & 3-L Transformer & & & 0.24 & 0.44 & 0.27 \\
\hline & & & & & $\checkmark$ & 6-L Transformer & & & 0.24 & 0.46 & 0.28 \\
\hline & & $\checkmark$ & & & & Passthrough & \multirow{4}{*}{$\begin{array}{c}\text { 6-Layer } \\
\text { Transformer }\end{array}$} & \multirow{4}{*}{ Beamsearch } & 0.22 & 0.40 & 0.22 \\
\hline & & $\checkmark$ & & & & 1-L Transformer & & & 0.21 & 0.41 & 0.26 \\
\hline & & $\checkmark$ & & & & 3-L Transformer & & & 0.22 & 0.41 & 0.22 \\
\hline & & $\checkmark$ & & & & 6-L Transformer & & & 0.23 & 0.45 & 0.27 \\
\hline$\checkmark$ & $\checkmark$ & $\checkmark$ & & & & Passthrough & \multirow{4}{*}{$\begin{array}{c}\text { 6-Layer } \\
\text { Transformer }\end{array}$} & \multirow{4}{*}{ Beamsearch } & 0.09 & 0.27 & 0.09 \\
\hline$\checkmark$ & $\checkmark$ & $\checkmark$ & & & & 1-L Transformer & & & 0.24 & 0.43 & 0.24 \\
\hline$\checkmark$ & $\checkmark$ & $\checkmark$ & & & & 3-L Transformer & & & 0.22 & 0.42 & 0.26 \\
\hline \multirow[t]{7}{*}{$\checkmark$} & $\checkmark$ & $\checkmark$ & & & & 6-L Transformer & & & 0.22 & 0.44 & 0.22 \\
\hline & & & & & $\checkmark$ & \multirow{3}{*}{$\begin{array}{c}\text { 6-Layer } \\
\text { Transformer }\end{array}$} & 1-L Transformer & \multirow{3}{*}{ Beamsearch } & 0.22 & 0.42 & 0.25 \\
\hline & & & & & $\checkmark$ & & 3-L Transformer & & 0.23 & 0.42 & 0.25 \\
\hline & & & & & $\checkmark$ & & 6-L Transformer & & 0.24 & 0.46 & 0.28 \\
\hline & & & & & $\checkmark$ & \multirow{3}{*}{$\begin{array}{c}\text { 6-Layer } \\
\text { Transformer }\end{array}$} & \multirow{3}{*}{$\begin{array}{c}\text { 6-Layer } \\
\text { Transformer }\end{array}$} & Greedy & 0.21 & 0.44 & 0.18 \\
\hline & & & & & $\checkmark$ & & & Multinomial & 0.20 & 0.42 & 0.16 \\
\hline & & & & & $\checkmark$ & & & Beamsearch & 0.24 & 0.46 & 0.28 \\
\hline
\end{tabular}

Table 3: Variants and ablations for the Neural Naturalist model. We find the best performing combination is an elementwise multiplication $(\odot)$ for the joint encoding, a 6-layer Transformer comparative module, a 6-layer Transformer decoder, and using beamsearch to perform inference.

the following section, we split an analysis of the model into two parts: largely positive findings, as well as common error cases.

\section{Positive Findings}

We find that the model exhibits dynamic granularity, by which we mean that it adjusts the magnitude of the descriptions based on the scale of differences between the two animals. If two animals are quite similar, it generates fine-grained descriptions such as, "Animal 2 has a slightly more curved beak than Animal 1," or "Animal 1 is more iridescent than Animal 2." If instead the two animals are very different, it will generate text describing larger-scale differences, like, "Animal 1 has a much longer neck than Animal 2," or "Animal 1 is mostly white with a black head. Animal 2 is almost completely yellow."

We also observe that the model is able to pro- duce coherent paragraphs of varying linguistic structure. These include a range of comparisons set up across both single and multiple sentences. For example, one it generates straightforward comparisons of the form, Animal 1 has $X$, while Animal 2 has $Y$. But it also generates contrastive expressions with longer dependencies, such as Animal 1 is $X, Y$, and $Z$. Animal 2 is very similar, except $W$. Furthermore, the model will mix and match different comparative structures within a single paragraph.

Finally, in addition to varying linguistic structure, we find the model is able to produce coherent semantics through a series of statements. For example, consider the following full output: "Animal 1 has a very long neck compared to Animal 2. Animal 1 has shorter legs than Animal 2. Animal 1 has a black beak, Animal 2 has a brown beak. Animal 1 has a yellow belly. Animal 2 has 


\begin{tabular}{lrrrrrr}
\hline & VISUAL & SPECIES & GENUS & FAMILY & ORDER & CLASS \\
\hline Freq. & 0.00 & 0.00 & 0.00 & 0.00 & 0.00 & 0.00 \\
Text-Only & 0.00 & -0.10 & -0.05 & 0.00 & 0.15 & -0.15 \\
CNN + LSTM & -0.15 & $\mathbf{0 . 2 0}$ & 0.15 & $\mathbf{0 . 5 0}$ & 0.40 & 0.15 \\
CNN + Attn. + LSTM & $\mathbf{0 . 1 5}$ & 0.15 & 0.15 & -0.05 & 0.05 & 0.20 \\
Neural Naturalist & 0.10 & -0.10 & $\mathbf{0 . 3 5}$ & 0.40 & $\mathbf{0 . 4 5}$ & $\mathbf{0 . 5 5}$ \\
\hline Human & 0.55 & 0.55 & 0.85 & 1.00 & 1.00 & 1.00 \\
\hline
\end{tabular}

Table 4: Human evaluation results on 120 test set samples, twenty per column. Scale: -1 (perfectly wrong) to 1 (perfectly correct). Columns are ordered left-toright by increasing distance. Our model outperforms baselines for several distances, though highly similar comparisons still prove difficult.

darker wings than Animal 1." The range of concepts in the output covers neck, legs, beak, belly, wings without repeating any topic or getting sidetracked.

\section{Error Analysis}

We also observe several patterns in the model's shortcomings. The most prominent error case is that the model will sometimes hallucinate differences (Figure 5, bottom row). These range from pointing out significant changes that are missing (e.g., "a black head" where there is none (Fig. 5, bottom left)), to clawing at subtle distinctions where there are none (e.g., "[its] colors are brighter ... and [it] is a bit bigger" (Fig. 5, bottom right)). We suspect that the model has learned some associations between common features in animals, and will sometimes favor these associations over visual evidence.

The second common error case is missing obvious distinctions. This is observed in Fig. 5 (bottom middle), where the prominent beak of Animal 1 is ignored by the model in favor of mundane details. While outlying features make for lively descriptions, we hypothesize that the model may sometimes avoid taking them into account given its per-token cross entropy learning objective.

Finally, we also observe the model sometimes swaps which features are attributed to which animal. This is partially observed in Fig. 5 (bottom left), where the "black head" actually belongs to Animal 1, not Animal 2. We suspect that mixing up references may be a trade-off for the representational power of attending over both images; there is no explicit bookkeeping mechanism to enforce which phrases refer to which feature comparisons in each image.

\section{Related Work}

Employing visual comparisons to elicit focused natural language observations was proposed by (Maji, 2012), and later investigated in the context of crowdsourcing by (Zou et al., 2015). We take inspiration from these works.

Previous work has collected natural language captions of bird photographs: CUB Captions (Reed et al., 2016) and CUB-Justify (Vedantam et al., 2017) are both language annotations on top of the CUB-2011 dataset of bird photographs (Wah et al., 2011). In addition to describing two photos instead of one, the language in our dataset is more complex by comparison, containing a diversity of comparative structures and implied semantics. We also collect our data without an anatomical guide for annotators, yielding everyday language in place of scientific terminology.

Conceptually, our paper offers a complementary approach to works that generate single-image class-discriminative or image-discriminative captions (Hendricks et al., 2016; Vedantam et al., 2017). Rather than discriminative captioning, we focus on comparative language as a means for bridging the gap between varying granularities of visual diversity.

Methodologically, our work is most closely related to the Spot-the-diff dataset (Jhamtani and Berg-Kirkpatrick, 2018). While dataset captions two images with only a small section of pixels that change (surveillance footage), we consider image pairs with no pixel overlap, which motivates our stratified sampling approach for drawing good comparisons.

Finally, the recently released $\mathrm{NLVR}^{2}$ dataset (Suhr et al., 2018) introduces a challenging natural language reasoning task using two images as context. Our work instead focuses on generating comparative language rather than reasoning.

\section{Conclusion}

We present the new Birds-to-Words dataset and Neural Naturalist model for generating comparative explanations of fine-grained visual differences. This dataset features paragraph-length, adaptively detailed descriptions written in everyday language. We hope that continued study of this area will produce models that can aid humans in critical domains like citizen science. 


\section{References}

Jia Deng, Wei Dong, Richard Socher, Li-Jia Li, Kai Li, and Li Fei-Fei. 2009. Imagenet: A large-scale hierarchical image database.

Ruiqi Guo, Sanjiv Kumar, Krzysztof Choromanski, and David Simcha. 2016. Quantization based fast inner product search. In Artificial Intelligence and Statistics, pages 482-490.

Kaiming He, Xiangyu Zhang, Shaoqing Ren, and Jian Sun. 2016. Deep residual learning for image recognition. In Proceedings of the IEEE conference on computer vision and pattern recognition, pages 770 778.

Lisa Anne Hendricks, Zeynep Akata, Marcus Rohrbach, Jeff Donahue, Bernt Schiele, and Trevor Darrell. 2016. Generating visual explanations. In European Conference on Computer Vision, pages 3-19. Springer.

Harsh Jhamtani and Taylor Berg-Kirkpatrick. 2018. Learning to describe differences between pairs of similar images. arXiv preprint arXiv:1808.10584.

Jonathan Krause, Michael Stark, Jia Deng, and Li FeiFei. 2013. 3d object representations for fine-grained categorization. In 4th International IEEE Workshop on 3D Representation and Recognition (3dRR-13), Sydney, Australia.

Chin-Yew Lin. 2004. Rouge: A package for automatic evaluation of summaries. Text Summarization Branches Out.

Subhransu Maji. 2012. Discovering a lexicon of parts and attributes. In European Conference on Computer Vision, pages 21-30. Springer.

Tomáš Mikolov, Martin Karafiát, Lukáš Burget, Jan Černockỳ, and Sanjeev Khudanpur. 2010. Recurrent neural network based language model. In Eleventh annual conference of the international speech communication association.

Erik Murphy-Chutorian and Mohan Manubhai Trivedi. 2009. Head pose estimation in computer vision: A survey. IEEE transactions on pattern analysis and machine intelligence, 31(4):607-626.

Kishore Papineni, Salim Roukos, Todd Ward, and WeiJing Zhu. 2002. Bleu: a method for automatic evaluation of machine translation. In Proceedings of the 40th annual meeting on association for computational linguistics, pages 311-318. Association for Computational Linguistics.

Scott Reed, Zeynep Akata, Honglak Lee, and Bernt Schiele. 2016. Learning deep representations of fine-grained visual descriptions. In Proceedings of the IEEE Conference on Computer Vision and Pattern Recognition, pages 49-58.
Piyush Sharma, Nan Ding, Sebastian Goodman, and Radu Soricut. 2018. Conceptual captions: A cleaned, hypernymed, image alt-text dataset for automatic image captioning. In Proceedings of the 56th Annual Meeting of the Association for Computational Linguistics (Volume 1: Long Papers), volume 1, pages 2556-2565.

Alane Suhr, Stephanie Zhou, Iris Zhang, Huajun Bai, and Yoav Artzi. 2018. A corpus for reasoning about natural language grounded in photographs. CoRR, abs/1811.00491.

Christian Szegedy, Sergey Ioffe, Vincent Vanhoucke, and Alexander A Alemi. 2017. Inception-v4, inception-resnet and the impact of residual connections on learning. In Thirty-First AAAI Conference on Artificial Intelligence.

Ashish Vaswani, Noam Shazeer, Niki Parmar, Jakob Uszkoreit, Llion Jones, Aidan N Gomez, Łukasz Kaiser, and Illia Polosukhin. 2017. Attention is all you need. In Advances in Neural Information Processing Systems, pages 5998-6008.

Ramakrishna Vedantam, Samy Bengio, Kevin Murphy, Devi Parikh, and Gal Chechik. 2017. Context-aware captions from context-agnostic supervision. In Proceedings of the IEEE Conference on Computer Vision and Pattern Recognition, pages 251-260.

Ramakrishna Vedantam, C Lawrence Zitnick, and Devi Parikh. 2015. Cider: Consensus-based image description evaluation. In Proceedings of the IEEE conference on computer vision and pattern recognition, pages 4566-4575.

Oriol Vinyals, Alexander Toshev, Samy Bengio, and Dumitru Erhan. 2015. Show and tell: A neural image caption generator. In Proceedings of the IEEE conference on computer vision and pattern recognition, pages 3156-3164.

Catherine Wah, Steve Branson, Peter Welinder, Pietro Perona, and Serge Belongie. 2011. The caltech-ucsd birds-200-2011 dataset.

Xiang Wu, Ruiqi Guo, Ananda Theertha Suresh, Sanjiv Kumar, Daniel N Holtmann-Rice, David Simcha, and Felix Yu. 2017. Multiscale quantization for fast similarity search. In Advances in Neural Information Processing Systems, pages 5745-5755.

Kelvin Xu, Jimmy Ba, Ryan Kiros, Kyunghyun Cho, Aaron Courville, Ruslan Salakhudinov, Rich Zemel, and Yoshua Bengio. 2015. Show, attend and tell: Neural image caption generation with visual attention. In International conference on machine learning, pages 2048-2057.

James Y Zou, Kamalika Chaudhuri, and Adam Tauman Kalai. 2015. Crowdsourcing feature discovery via adaptively chosen comparisons. arXiv preprint arXiv:1504.00064. 